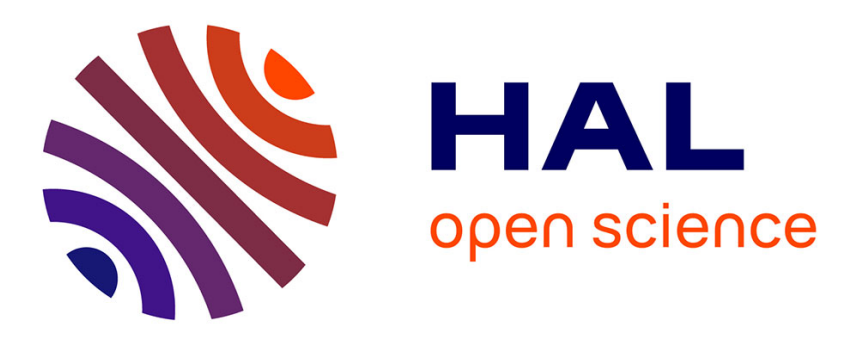

\title{
Surveillance épidémiologique des troubles musculo-squelettiques du membre supérieur en entreprises dans l'Ouest algérien
}

Omar Ghomari, Benali Beghdadli, Mourad Taleb, Abdelkader Baderdine Kandouci, Alexis Descatha, Yves Roquelaure, Serge Fanello

\section{To cite this version:}

Omar Ghomari, Benali Beghdadli, Mourad Taleb, Abdelkader Baderdine Kandouci, Alexis Descatha, et al.. Surveillance épidémiologique des troubles musculo-squelettiques du membre supérieur en entreprises dans l'Ouest algérien. Archives des Maladies Professionnelles et de L'Environnement, 2010, 71 (5), pp.781-789. 10.1016/j.admp.2010.06.007 . hal-03389578

\section{HAL Id: hal-03389578 \\ https://univ-angers.hal.science/hal-03389578}

Submitted on 21 Oct 2021

HAL is a multi-disciplinary open access archive for the deposit and dissemination of scientific research documents, whether they are published or not. The documents may come from teaching and research institutions in France or abroad, or from public or private research centers.
L'archive ouverte pluridisciplinaire $\mathbf{H A L}$, est destinée au dépôt et à la diffusion de documents scientifiques de niveau recherche, publiés ou non, émanant des établissements d'enseignement et de recherche français ou étrangers, des laboratoires publics ou privés. 


\title{
Surveillance épidémiologique des troubles musculo-squelettiques du membre supérieur en entreprises dans l'Ouest algérien
}

\author{
Epidemiological surveillance of upper limb musculoskeletal \\ disorders in occupational setting in western Algeria
}

\author{
O. Ghomari ${ }^{a}$, B. Beghdadli ${ }^{a}$, M. Taleb ${ }^{a}$, A.B. Kandouci ${ }^{a}$, A. Descatha ${ }^{\text {, }}$ \\ Y. Roquelaurec, S. Fanello ${ }^{\mathrm{d} *}$
}

a Laboratoire de recherche environnement et santé, université de Sidi bel Abbès, 14, rue Boukhari Larbi, 22000 Sidi bel Abbès, Algérie

${ }^{\mathrm{b}}$ Unité de pathologie professionnelle, UVSO Inserm U687, groupe hospitalier RaymondPoincaré, hôpital maritime de Berck, 104, boulevard Raymond-Poincaré, 92380 Garches, France

' Laboratoire d'épidémiologie et d'ergonomie en santé et travail, UFR médecine Angers, 2, rue Haute-de-Reculée, 49045 Angers cedex, France

Disponible en ligne sur ScienceDirect www.sciencedirect.com

49933 Angers cedex 9, France

\section{Summary}

This cross-sectional and multicenter study aims to estimate the prevalence of upper limb musculoskeletal disorders (ULMSDs) and exposure to risk factors in industrial companies, through occupational physicians organized in an epidemiological sentinel surveillance network.

Methods. In 2008, clinical data were collected using a questionnaire of the "Nordic" type for morbidity symptoms and clinical examination, according to the standardized diagnostic approach of the European consensus SALTSA. ULMSDs were classified into three degrees of severity after validation of the diagnosis via a decision tree. Data on risk factors for ULMSDs were collected by a questionnaire including the biomechanical, psychosocial and organizational factors. Exposure scores were calculated for each anatomical area of the upper limb by addition of the risk factors revealed by the consensus.

Results. Thus, 933 employees in eight industrial companies were included at random. Almost one in two workers in the past 12 months and one in four in the past seven days have suffered from non-specific upper limb musculoskeletal symptoms. The prevalence of diagnosed ULMSDs was high: $12 \%$ of the workers have sustained at least one proven form of unilateral or bilateral ULMSD, $6.3 \%$ a rotator cuff syndrome, $1.8 \%$ had lateral epicondylitis, $2.8 \%$ a carpal tunnel syndrome. The prevalence of ULMSDs increased with age and varied

\section{Résumé}

Cette étude transversale et multicentrique a pour objectif d'estimer la prévalence des troubles musculo-squelettiques.du membre supérieur (TMSms) et l'exposition aux facteurs de risque grâce à des médecins du travail sentinelles organisés en réseau de surveillance épidémiologique au niveau des entreprises.

Méthodes. En 2008, les données cliniques ont été recueillies à l'aide du questionnaire « Nordic » et d'un examen clinique standardisé, selon la démarche diagnostique du consensus européen Saltsa. Les TMSms ont été classés en trois stades de gravité après validation d'un arbre diagnostique. Les facteurs de risque des TMSms ont été recensés par questionnaire comprenant les facteurs biomécaniques, psychosociaux et organisationnels. Des scores d'exposition ont été calculés pour chaque zone anatomique du membre supérieur par addition des facteurs de risque pris en compte par le consensus.

Résultats. Ainsi, 933 salariés appartenant à huit entreprises industrielles ont été inclus par tirage au sort. Près d'un salarié sur deux et un salarié sur quatre ont souffert au cours des 12 derniers mois et les sept derniers jours de symptômes musculo-squelettiques. La prévalence des TMS diagnostiqués était élevée : $12 \%$ des salariés ont présenté au moins une forme avérée unilatérale et/ou bilatérale de TMSms, 6,3\% un syndrome de la coiffe des rotateurs, 1,8\% une épicondylite latérale, 2,8 \% un syndrome du canal carpien. La prévalence des symptômes et des TMSms avérés augmentait avec

* Auteur correspondant.

e-mail : sefanello@chu-angers.fr

1775-8785X/\$ - see front matter @ $\odot 2010$ Elsevier Masson SAS. Tous droits réservés.

10.1016/j.admp.2010.06.007 Archives des Maladies Professionnelles et de l'Environnement 2010;71:781-789 
widely across companies and occupations. One in two workers was exposed to at least two risk factors for the upper limb, warning threshold of the SALTSA consensus.

Conclusion. The use of the SALTSA protocol for the first time in Algerian companies revealed the importance of the prevalence of ULMSDs and of exposure to risk factors. These results show the need to structure the occupational physicians into an epidemiological sentinel surveillance network so as to develop a prevention program in most sectors and reduce the prevalence of ULMSDs.

(c) 2010 Elsevier Masson SAS. All rights reserved.

Keywords: Musculoskeletal disorders, Prevalence, Risk factors

\section{Introduction}

Les troubles musculo-squelettiques des membres supérieurs (TMSms) regroupent un ensemble d'affections péri-articulaires touchant les tissus mous suite à une hypersollicitation d'origine professionnelle du membre supérieur (MS) survenant chez des travailleurs [1]. Ils constituent une préoccupation majeure en santé au travail de part leur augmentation constante et l'importance du retentissement socioprofessionnel en termes de coût humain et de douleurs. Les TMSms reconnus comme maladies professionnelles (MP) occupent la première place au niveau de l'union européenne [2]. En Algérie, les TMSms n'apparaissent pas encore comme un réel problème de santé au travail, bien qu'une étude récente ait rapporté une prévalence élevée [3].

Le but de ce travail était d'estimer la prévalence des TMSms et l'intensité de l'exposition aux facteurs de risque professionnels en milieu industriel, selon une méthodologie éprouvée [4-6].

\section{Sujets et méthodes}

Cette enquête, multicentrique, descriptive, transversale, menée en 2008, a intéressé les salariés de huit entreprises industrielles, publiques et privées de la ville de Sidi bel Abbès appartenant aux secteurs manufacturier et agroalimentaire, programmés pour la visite périodique médicale durant la période de l'enquête.

\section{Population étudiée}

L'enquête a concerné un échantillon aléatoire de salariés, tiré au sort sur une période de six mois en respectant la proportionnalité par entreprise. Les salariés inclus étaient âgés de 20 à 59 ans, quel que soit le type du contrat de travail, l'âge et variait largement en fonction des entreprises et des professions. Un salarié sur deux était exposé à au moins deux facteurs de risque au niveau du membre supérieur, seuil d'alerte du consensus Saltsa.

Conclusion. L'utilisation du protocole Saltsa pour la première fois dans des entreprises algériennes a révélé l'importance de la prévalence des TMS et de l'exposition aux facteurs de risques. Ces résultats montrent la nécessité de la structuration des médecins du travail en réseau de surveillance épidémiologique afin d'élaborer un programme de prévention dans la plupart des secteurs pour réduire la prévalence des TMSms.

(C) 2010 Elsevier Masson SAS. Tous droits réservés.

Mots clés : Troubles musculo-squelettiques, Prévalence, Facteurs de risque

souffrant ou non d'affections ostéo-articulaires des MS avec une ancienneté de plus de un an au poste actuel, sous réserve de leur accord de participation.

\section{Modalités de recueil de données}

Préalablement à la mise en place du réseau, les autorisations de la direction de la santé et des entreprises ont été obtenues. Onze médecins volontaires ont participé à ce réseau et avaient tous reçu une formation à la démarche du consensus européen Saltsa (examen standardisé) l'outil Saltsa est orienté vers la recherche de troubles infracliniques et cliniques et vise à déceler des signes " sentinelles " précoces des principaux TMSms pour en évaluer l'incidence et la prévalence dans une optique de prévention $[4,5]$ ) et au protocole de la surveillance [6]. Au cours des visites médicales périodiques, le médecin interrogeait le salarié sur les différents items du questionnaire en français et en arabe dialectal préalablement homogénéisés pour l'ensemble des médecins du réseau. La première partie inspirée du questionnaire " Nordic " permettait le recueil des symptômes musculosquelettiques (SMS), sur les 12 derniers mois et les sept derniers jours, au niveau des différentes zones anatomiques du membre supérieur (MS) et le cou $[6,7]$. L'examen clinique standardisé était réalisé en cas de plaintes récentes. Les TMSms retenus étaient les quatre principaux types de tendinopathies: le syndrome de la coiffe des rotateurs de l'épaule (SCR), l'épicondylite latérale (EPL), les tendinites des fléchisseurs ou des extenseurs du poignet et des doigts (TEF), la ténosynovite de De Quervain (TDO) et les deux principaux syndromes canalaires, syndrome du canal carpien (SCC), syndrome du tunnel ulnaire ou cubital (STC) au niveau de la gouttière épitrochléo-olécrânienne. Les données de l'exposition professionnelle aux facteurs de risque de TMSms recueillies par questionnaires selon les critères du consensus 
Saltsa $[5,8]$, reprenaient les facteurs de risque biomécaniques et psychosociaux.

\section{Analyse des données}

Les questionnaires ont été saisis et traités par le logiciel SPSS 11.5. Le seuil de significativité a été fixé à cinq pour cent. Les analyses statistiques ont été réalisées séparément pour les deux sexes. L'analyse des données quantitatives a reposé sur l'étude de la variance et sur le test $(t)$ de Student pour la comparaison des moyennes. Les tests du $\boldsymbol{\chi}^{2}$ de Pearson, $\boldsymbol{\chi}^{2}$ de tendance ont été utilisés pour les grandeurs qualitatives. Des comparaisons de proportions ont été utilisées pour la représentativité de l'échantillon.

La prévalence des SMS et des TMSms diagnostiqués a été étudiée pour chaque localisation anatomique et pour l'ensemble du membre supérieur (cou exclu). Les TMSms ont été regroupés en six principaux TMSms (6TMS) et ont été ventilés en fonction des caractéristiques sociodémographiques et des secteurs industriels. Des scores d'exposition aux facteurs de risque ont été calculés, comptabilisant pour chaque localisation anatomique les facteurs biomécaniques et psychosociaux des TMS décrits dans le consensus (tableau I). L'exposition professionnelle était classée faible "zone verte ", modérée "zone jaune " et élevée "zone rouge " selon que le salarié était respectivement exposé à aucun, à un ou à au moins deux facteurs de risque. L'exposition du membre supérieur (cou excepté) était classée en "zone rouge " lorsque l'exposition au risque de TMS était classée en "zone rouge " pour au moins une des trois zones allant de l'épaule au poignet.

\section{Résultats}

\section{Caractéristiques sociodémographiques et professionnelles de la population}

Au total, 933 salariés ont été inclus dans notre échantillon parmi une population totale de 3630 salariés. Le sexe masculin représentait $54,2 \%$ avec un âge moyen de $42,1 \pm 0,34$ ans. Les femmes étaient en moyenne moins âgées, 40,6 10,39 ans $(p<0,01)$. Les quadragénaires représentaient la moitié des effectifs devant les trentenaires et les quinquagénaires. La catégorie ouvrière qualifiée ou non était la plus représentée. Plus d'un salarié sur deux avaient une ancienneté de plus de dix ans. Trois salariés sur quatre avaient un contrat de travail de type permanent. L'échantillon de salariés était représentatif de la population de référence sur l'âge, le sexe, l'ancienneté et les catégories professionnelles (tableau II).

\section{Prévalence des symptômes musculo-squelettiques}

La prévalence des SMS au cours des 12 derniers mois était très élevée pour les salariés des deux sexes. Près de $45 \%$ des hommes et $55 \%$ des femmes ont rapporté des plaintes au niveau du membre supérieur, dans au moins une des zones anatomiques suivantes : épaule, coude, poignet et le cou. Les femmes ont rapporté plus de SMS, au niveau du membre supérieur $(p<0,05)$, des épaules $(p<0,001)$, et des poignets $(p<0,05)$. Les plaintes au niveau de la région du coude/avant bras étaient plus élevées chez les hommes $(p<0,05)$.

Tableau I

Définition des facteurs de risque généraux et posturaux spécifiques des TMSms selon le consensus Saltsa.

\section{Facteurs de risque}

Répétitivité

Force

Absence de récupération

Demande psychologique élevée

Soutien social faible

Postures extrêmesNuque et cou

Postures extrêmesÉpaule et bras

Postures extrêmesCoude et avant-bras

Postures extrêmesPoignet et main

\section{Critères de définition}

Mouvements très répétitifs du membre supérieur plus de 4 heures par jour Manipulation de charges de plus de $4 \mathrm{~kg}$ plus de 4 heures par jour

Pauses $<10$ minutes par heure si les gestes sont très répétitifs

Score $\geq 75 \%$ du maximum

Score $\leq \mathbf{2 5} \%$ du maximum

Mouvements de flexion du cou plus de 4 heures par jour Mouvements d'extension du cou plus de 4 heures par jour Travail bras éloignés du corps plus de 4 heures par jour Travail sur écran ou binoculaire plus de 4 heures par jour

Travail mains au-dessus des épaules plus de 2 heures par jour Extension du ou des bras en arrière plus de 2 heures par jour Travail bras éloignés du corps plus de 2 heures par jour

Mouvement de flexion/extension du coude plus de 2 heures par jour Mouvement de prono supination plus de 2 heures par jour

Mouvement de torsion du poignet plus de 2 heures par jour Utilisation de la pince pouce-index plus de 4 heures par jour Utilisation d'un outil vibrant plus de 1 heure par jour Utilisation d'un clavier/souris informatique plus de 4 heures par jour 


\begin{tabular}{|c|c|c|c|c|c|}
\hline \multirow[t]{2}{*}{ Caractéristiques } & \multicolumn{2}{|c|}{ Échantillon ( $n=933)$} & \multicolumn{2}{|c|}{$\begin{array}{l}\text { Population référence } \\
(n=3630)\end{array}$} & \multirow[t]{2}{*}{$p$-valeur } \\
\hline & $n$ & $\%$ & $n$ & $\%$ & \\
\hline \multicolumn{6}{|l|}{$\hat{A} g e$} \\
\hline $20-29$ & 90 & 9,6 & 405 & 11,1 & 0,13 \\
\hline $30-39$ & 222 & 23,8 & 875 & 24,1 & 0,38 \\
\hline $40-49$ & 479 & 51,3 & 1756 & 48,5 & 0,09 \\
\hline $50-59$ & 142 & 15,2 & 594 & 16,3 & 0,26 \\
\hline \multicolumn{6}{|l|}{ Sexe } \\
\hline Masculin & 506 & 54,2 & 1839 & 50,6 & 0,17 \\
\hline Féminin & 427 & 45,8 & 1791 & 49,3 & 0,18 \\
\hline \multicolumn{6}{|l|}{ Profession } \\
\hline Ouvrier non qualifié & 517 & 55,4 & 2124 & 58,5 & 0,06 \\
\hline Ouvriers qualifiés & 225 & 24,1 & 891 & 27,1 & 0,051 \\
\hline Agent de maîtrise & 46 & 5,0 & 104 & 6,1 & 0,17 \\
\hline Technicien & 35 & 3,8 & 115 & 3,1 & 0,18 \\
\hline Cadre et ingénieur & 19 & 2,0 & 121 & 3,3 & 0,033 \\
\hline Employé & 88 & 9,4 & 275 & 7,5 & 0,039 \\
\hline \multicolumn{6}{|l|}{ Ancienneté } \\
\hline$\leq 10$ ans & 416 & 44,6 & 1503 & 41,4 & 0,055 \\
\hline$>10$ ans & 517 & 55,4 & 2127 & 58,6 & 0,056 \\
\hline \multicolumn{6}{|l|}{ Statut de l'emploi } \\
\hline CDI & 718 & 77 & 2687 & 74,0 & 0,044 \\
\hline CDD & 215 & 23 & 943 & 25,9 & 0,051 \\
\hline
\end{tabular}

$C D I$ : contrat à durée indéterminée; $C D D$ : contrat à durée déterminée.

Au cours des sept derniers jours, les femmes ont aussi souffert plus, au niveau du membre supérieur ( $p<0,001)$, du cou $(p<0,05)$, de l'épaule et des poignets $(p<0,001)$. Parmi les salariés souffrants de SMS sur l'année écoulée, chez un homme sur trois et une femme sur deux environ, les symptômes ont duré au moins 30 jours au niveau du membre supérieur. La tendance à la chronicité des symptômes était aussi plus marquée chez les femmes au niveau du cou $(p<0,01)$ et des épaules $(p<0,01)$, de même que pour les symptômes permanents au niveau du membre supérieur $(p<0,05)$, du cou $(p<0,05)$ et de l'épaule $(p<0,05)$ (tableau III).

La prévalence des SMS au niveau du membre supérieur augmentait significativement avec l'âge chez les deux sexes. Les salariés quinquagénaires des deux sexes étaient les plus touchés.

La prévalence des SMS était élevée pratiquement dans toutes les entreprises et variait de manière significative $(p<0,001)$. Les hommes se plaignaient plus au niveau de la laiterie, l'abattoir de volaille, l'unité de maintenance ferroviaire, l'unité d'appareillage d'orthopédie. Pour les femmes, c'était l'industrie électronique et de l'habillement. La prévalence des SMS du membre supérieur était inversement proportionnelle au degré de qualification professionnelle. La catégorie ouvrière non qualifiée était la plus touchée par les SMS sur les 12 derniers mois et les sept derniers jours. La prévalence des SMS du membre supérieur et du cou augmentait de manière proportionnelle à l'ancienneté au poste de travail actuel aussi bien chez les hommes que chez les femmes.

\section{Prévalence des troubles musculo-squelettiques diagnostiqués}

Au total, 124 cas ont été diagnostiqués chez 110 salariés avec une prévalence de $11,8 \%$. Le SCR était l'affection la plus fréquente devant le SCC et I'EPL. Les femmes ont présenté plus de TMS au niveau du membre supérieur $(p<0,01)$, de l'épaule $(p<0,01)$ et du poignet $(p<0,05)$. La proportion de sujet souffrant d'au moins un des six TMSms augmentait avec l'âge dans les deux sexes. Après 50 ans, un salarié sur quatre présentait au moins un des six TMSms, trois femmes sur dix, et un homme sur cinq. Les femmes après 40 ans avaient significativement plus de TMSms que les hommes $(p<0,05)$ (tableau IV).

Les TMSms avérés étaient plus importants au niveau de l'industrie électronique et de l'habillement particulièrement chez les femmes. La proportion de sujet souffrant d'au moins un des six TMSms évoluait de manière proportionnelle à l'ancienneté dans le poste actuel chez les femmes $(p<0,001)$, mais pas chez les hommes $(p=0,19)$. Les ouvriers qualifiés ou non étaient les plus touchés par les TMS aussi bien chez les hommes que chez les femmes. 
Tableau III

Prévalence des symptômes musculo-squelettiques en fonction des zones anatomique et du sexe.

\begin{tabular}{|c|c|c|c|c|c|c|}
\hline Prévalence (\%) & & $\begin{array}{l}\text { Cou } \\
\% \text { [IC 95\%] }\end{array}$ & $\begin{array}{l}\text { Épaule/bras } \\
\% \text { [IC } 95 \%]\end{array}$ & $\begin{array}{l}\text { Coude/Avant-bras } \\
\% \text { [IC 95\%] }\end{array}$ & $\begin{array}{l}\text { Poignet/Main } \\
\% \text { [IC } 95 \% \text { ] }\end{array}$ & $\begin{array}{l}\text { Membre supérieur } \\
\% \text { [IC 95\%] }\end{array}$ \\
\hline $\begin{array}{l}\text { Symptômes au cours des } \\
12 \text { derniers mois }\end{array}$ & $\begin{array}{l}\text { Hommes } \\
\text { Femmes }\end{array}$ & $\begin{array}{l}17,6[14,0-21,2] \\
22,2[18,3-6,1]\end{array}$ & $\begin{array}{r}25,7[21,9-29,5] \\
37,7[33,1-42,3]\end{array}$ & $\begin{array}{l}14,8[11,7-7,9] \\
9,6[6,8-12,4]\end{array}$ & $\begin{array}{c}8,7[6,2-11,2] \\
15,0[11,6-18,4]\end{array}$ & $\begin{array}{l}45,3[40,6-50,0] \\
55,0[50,7-59,3]\end{array}$ \\
\hline $\begin{array}{l}\text { Symptômes pendant un } \\
\text { mois ou plus }{ }^{1}\end{array}$ & $\begin{array}{l}\text { Hommes } \\
\text { Femmes }\end{array}$ & $\begin{array}{l}7,0[3,7-10,3] \\
17[12,2-1,8]\end{array}$ & $\begin{array}{r}14,8[10,2-19,4] \\
27,2[21,5-32,9]\end{array}$ & $\begin{array}{l}9,6[5,8-13,4] \\
4,7[2,0-7,4]\end{array}$ & $\begin{array}{r}9,2[5,5-12,9] \\
10,6\left[6,7^{-14,5}\right]\end{array}$ & $\begin{array}{l}31,9[27,8-36,0] \\
47,2[42,9-51,9]\end{array}$ \\
\hline Symptômes quotidiens & $\begin{array}{l}\text { Hommes } \\
\text { Femmes }\end{array}$ & $\begin{array}{c}0,9[0,3-3,2] \\
5,5[2,6-8,4]\end{array}$ & $\begin{array}{l}1,7[0,7-4,3] \\
7,2[3,9-10,5]\end{array}$ & $\begin{array}{l}1,3[0,4-3,8] \\
1,7[0,8-4,3]\end{array}$ & $\begin{array}{l}2,6[1,2-5,6] \\
3,8[2,1-7,1]\end{array}$ & $\begin{array}{c}6,6[3,4-9,8] \\
14,5[10,0-19,0]\end{array}$ \\
\hline $\begin{array}{l}\text { Symptômes au cours } \\
\text { des } 7 \text { derniers jours }\end{array}$ & $\begin{array}{l}\text { Hommes } \\
\text { Femmes }\end{array}$ & $\begin{array}{c}6,1[4,0-8,2] \\
11,2[11,9-22,9]\end{array}$ & $\begin{array}{r}9,7[7,1-2,3] \\
21,1[17,2-5,0]\end{array}$ & $\begin{array}{l}5,9[3,8-8,0] \\
8,0[5,4-10,6]\end{array}$ & $\begin{array}{c}5,3[3,3-7,3] \\
12,4[9,3-15,5]\end{array}$ & $\begin{array}{r}18,6[15,2-22,0] \\
34,4[29,9-38,9]\end{array}$ \\
\hline
\end{tabular}

Un parmi les salariés ayant eu des symptômes durant les 12 derniers mois.

Tableau IV

Prévalence des TMSms diagnostiqués en fonction de l'âge. (les pathologies sont comptabilisées par sujet).

\begin{tabular}{|c|c|c|c|c|c|}
\hline Prévalence (\%) & $\begin{array}{l}20-29 \text { ans } \\
n \%[\text { IC } 95 \%]\end{array}$ & $\begin{array}{l}30-39 \text { ans } \\
n \%[\text { IC } 95 \%]\end{array}$ & $\begin{array}{l}40-49 \text { ans } \\
n \%[\text { IC } 95 \%]\end{array}$ & $\begin{array}{l}50-59 \text { ans } \\
n \%[\text { IC } 95 \%]\end{array}$ & $\begin{array}{l}\text { Ensemble } \\
n \text { \% [IC } 95 \% \text { ] }\end{array}$ \\
\hline \multicolumn{6}{|l|}{ Hommes } \\
\hline Syndrome de la coiffe des rotateurs & $\circ \circ[0,0-0,0]$ & $43,2[1,7-4,7]$ & $83,1[1,6-4,6]$ & $78,3[5,9-10,7]$ & $193,8[2,1-5,5]$ \\
\hline Épicondylite latérale & $0 \circ[0,0-0,0]$ & $\circ \circ[0,0-0,0]$ & $41,6[0,8-3,1]$ & $67,1[4,9-9,3]$ & $102,0[0,8-3,2]$ \\
\hline Syndrome du tunnel cubital & $\circ \circ[0,0-0,0]$ & $\circ \circ[0,0-0,0]$ & $20,8[0,3-2,0]$ & $\circ \circ[0,0-0,0]$ & $20,4[0,1-1,4]$ \\
\hline Syndrome du canal carpien & $12,6[1,4-4,4]$ & $\circ \circ[0,0-0,0]$ & $41,6[0,8-3,1]$ & $33,6[2,0-5,2]$ & $81,6[0,8-3,1]$ \\
\hline Tendinite des poignets & $\circ \circ[0,0-0,0]$ & $10,8[0,3-2,0]$ & $10,4[0,1-1,4]$ & $11,2[0,6-2,6]$ & $30,6[0,2-1,7]$ \\
\hline Maladie de De Quervain & $0 \circ[0,0-0,0]$ & $\circ \circ[0,0-0,0]$ & $31,2[0,6-2,6]$ & 0 o, o [0,0-0,o] & $30,6[0,2-1,7]$ \\
\hline Avoir un des six TMSms & $12,6[1,4-4,4]$ & $54,0[2,3-5,7]$ & $228,5[6,1-10,9]$ & $1619,0[15,6-2,4]$ & $448,7[6,2-11,2]$ \\
\hline \multicolumn{6}{|l|}{ Femmes } \\
\hline Syndrome de la coiffe des rot & $11,9[1,0-3,7]$ & $22,1[1,1-3,9]$ & $2511,3[8,3-14,3]$ & $1220,7[16,9-24,5]$ & $409,4[6,6-12,2]$ \\
\hline Épicondylite latérale & $\circ \circ[0,0-0,0]$ & $\circ \circ[0,0-0,0]$ & $31,4[0,6-3,0]$ & $23,4[1,7-5,1]$ & $51,2\left[0,5^{-2,8]}\right.$ \\
\hline Syndrome du tunnel cubital & $\circ \circ[0,0-0,0]$ & $\circ \circ[0,0-0,0]$ & $20,9[0,3-2,3]$ & $117,0[0,8-3,4]$ & $30,7[0,2-2,0]$ \\
\hline Syndrome du canal carpien & $\circ \circ[0,0-0,0]$ & $44,2[2,3-6,1]$ & $94,1[2,2-6,0]$ & $58,6[5,9-11,3]$ & $184,2[2,3-6,1]$ \\
\hline Tendinite des poignets & $\circ \circ[0,0-0,0]$ & $\circ \circ[0,0-0,0]$ & $20,9[0,3-2,3]$ & $11,7[0,8-3,4]$ & $30,7[0,2-2,0]$ \\
\hline Maladie de De Quervain & $\circ \circ[0,0-0,0]$ & $\circ \circ[0,0-0,0]$ & $31,4[0,6-3,0]$ & $11,7[0,8-3,4]$ & $40,9[0,3-2,3]$ \\
\hline Avoir un des six TMSms & $11,9[1,0-3,7]$ & $55,2[3,1-7,3]$ & $4118,6[14,9-2,3]$ & $1932,8[28,3-37,3]$ & $6615,5[12,1-18,9]$ \\
\hline
\end{tabular}

$n$ : nombre de cas de TMS ; \% : prévalence ; [IC $95 \%]$ : intervalle de confiance.

\section{L'exposition aux facteurs de risque de troubles musculo-squelettiques des membres supérieurs}

Au niveau du membre supérieur l'exposition à au moins deux facteurs de risque biomécaniques et psychosociaux des TMSms était élevée et classée en "zone rouge " pour plus d'un salarié sur deux. L'exposition à au moins deux facteurs de risque a concerné plus de quatre salariés sur dix si l'on considère uniquement le cou et plus d'un salarié sur deux pour la région de l'épaule, le coude et le poignet. Les femmes étaient significativement plus exposées à au moins deux facteurs de risque au niveau de l'ensemble des zones anatomiques du membre supérieur y compris le cou $(p<0,001)$ (tableau V). En moyenne, les femmes étaient exposées à $3,3 \pm 1,9$ facteurs, les hommes $2,1 \pm 1,9$ facteurs, $(p<0,001)$ au niveau du membre supérieur. L'exposition était classée en " zone verte " pour seulement un salarié sur trois environ si l'on considère uniquement le cou et environ un salarié sur six pour le membre supérieur dans son ensemble (nuque exclue). L'exposition était considérée faible et classée en "zone jaune ", pour un salarié sur quatre si l'on considère uniquement les TMS du cou et $17 \%$ pour l'ensemble du membre supérieur (cou exclu). Elle varie entre 23 et $25 \%$ pour les zones de l'épaule, le coude et le poignet. Les salariés cumulaient un grand nombre de facteurs de risque de TMSms, puisque $57 \%$ d'entre eux étaient exposés à au moins trois facteurs de risque de TMSms, $43 \%$ à au moins quatre facteurs et $8 \%$ à au moins sept facteurs de risque sur les 17 facteurs de risque pris en compte.

L'intensité de l'exposition ne variait pas significativement avec l'âge chez les deux sexes quelle que soit la zone anatomique du membre supérieur. Les salariés les plus jeunes (2029 ans) étaient autant exposés que ceux plus âgés (5059 ans). La catégorie ouvrière qualifiée ou non était la plus exposée respectivement $79 \%$ et $74 \%$, chez les deux sexes. Au niveau des entreprises, l'intensité de l'exposition du membre 
Tableau V

Distribution des scores d'exposition aux facteurs de risque biomécaniques et psychosociaux de TMSms en fonction de la zone anatomique et du sexe.

\begin{tabular}{|c|c|c|c|c|c|c|c|c|c|c|c|c|c|c|c|c|c|c|}
\hline \multirow{3}{*}{$\begin{array}{l}\text { Score d'exposition au } \\
\text { risque de tms }\end{array}$} & \multicolumn{6}{|c|}{ Hommes } & \multicolumn{6}{|c|}{ Femmes } & \multicolumn{6}{|c|}{ Ensemble } \\
\hline & \multicolumn{2}{|l|}{0} & \multicolumn{2}{|l|}{1} & \multicolumn{2}{|c|}{2 ou +} & \multicolumn{2}{|l|}{0} & \multicolumn{2}{|l|}{1} & \multicolumn{2}{|l|}{2} & \multicolumn{2}{|l|}{0} & \multicolumn{2}{|l|}{1} & \multicolumn{2}{|c|}{2 ou +} \\
\hline & $n$ & $\%$ & $n$ & $\%$ & $n$ & $\%$ & $n$ & $\%$ & $\bar{n}$ & $\%$ & $n$ & $\%$ & $n$ & $\%$ & $n$ & $\%$ & $n$ & $\%$ \\
\hline $\mathrm{Cou}$ & 227 & 44,9 & 165 & 32,6 & 114 & 22,5 & 55 & 12,9 & 81 & 19 & 291 & 68,1 & 282 & 30,2 & 246 & 26,4 & 405 & 43,4 \\
\hline Épaule & 183 & 36,2 & 134 & 26,5 & 189 & 37,4 & 48 & 11,2 & 97 & 22,7 & 282 & 66 & 231 & 24,8 & 231 & 24,8 & 471 & 50,5 \\
\hline Coude & 165 & 32,6 & 125 & 24,7 & 216 & 42,7 & 53 & 12,4 & 96 & 22,5 & 278 & 65,1 & 218 & 23,4 & 221 & 23,7 & 494 & 52,9 \\
\hline Poignet & 164 & 32,4 & 131 & 25,9 & 211 & 41,7 & 53 & 12,4 & 63 & 14,8 & 311 & 72,8 & 217 & 23,3 & 194 & 20,8 & 522 & 55,9 \\
\hline Ensemble membre & 128 & 25,3 & 89 & 17,6 & 289 & 57,1 & 34 & 8,0 & 52 & 12,2 & 341 & 79,9 & 162 & 17,4 & 141 & 15,1 & 630 & 67,5 \\
\hline
\end{tabular}

supérieur

(nuque exclue)

supérieur (cou exclu) était importante. Elle variait entre $58 \%$ à $84 \%$. Le niveau d'exposition était particulièrement intense au niveau de l'industrie électronique, l'abattoir, le centre d'enfûtage et l'industrie de l'habillement.

La prévalence des SMS au cours des 12 derniers mois et les sept derniers jours augmentait significativement avec le nombre de facteurs de risque identifiés au poste de travail. Ainsi, celle des 12 derniers mois passait de $14 \%, 28 \%$ à $53 \%$ pour une exposition de $0 \%, 2 \%, 5 \%$ facteurs de risque respectivement ( $\chi^{2}$ de tendance, $p<0,001$ ), de même pour les SMS au cours des sept derniers jours ou la prévalence passait de 6 à $89 \%$ lorsque le nombre des facteurs de risque cumulés passait respectivement de zéro à huit et plus ( $\chi^{2}$ de tendance, $p<0,001$ ). La proportion de sujet souffrant d'au moins un des six TMSms augmentait significativement avec l'intensité de l'exposition. Elle passait de 2 à $44 \%$ lorsque le nombre de facteurs de risque passait de zéro à huit et plus $\left(\chi^{2}\right.$ de tendance $p<0,001)$.

\section{Discussion}

Dans notre étude, nous avons utilisé le même protocole que celui des pays de la Loire [6]. Le choix du type transversal dans notre étude a été motivé afin de démontrer la faisabilité du protocole de surveillance restreignant ainsi l'effectif des salariés. Notre étude s'est par ailleurs limitée à certaines entreprises relevant de certains secteurs industriels, car le service de médecine du travail ne surveillait que les entreprises conventionnées. Ces entreprises représentaient cependant à elles seules plus de $50 \%$ des entreprises de main d'œuvre associées classiquement à un risque élevé de TMSms.

L'échantillon de salariés était représentatif de la région, l'âge, le sexe, les catégories professionnelles et l'ancienneté au poste actuel. Les cadres étaient peu présents en visites périodiques par rapport aux employés, ce qui pourrait expliquer les différences observées. Cela n'a pas d'influence sur nos résultats car ces deux catégories ne présentaient pas de TMSms. La prédominance de la classe ouvrière doit être prise en consi- dération dans l'interprétation des résultats, car il s'agit d'une population à risque élevé de TMSms $[9,10]$.

\section{Recueil des données médicales}

Le questionnaire scandinave « Nordic " remanié par Hagberg [11], se rapporte aux 12 derniers mois et aux sept derniers jours, ce qui limite les biais de mémorisation [12] et offre une mesure sensible et reproductible de l'intensité et la fréquence des symptômes $[10,13]$. Néanmoins, il doit être complété par un examen clinique pour pouvoir établir un diagnostic précis [13]. La méthodologie d'examen clinique a été jugée très favorablement par les médecins du réseau, mais a nécessité une formation préalable afin d'homogénéiser les pratiques cliniques et d'assurer sa qualité et son objectivité [14].

\section{Prévalence des symptômes musculo-squelettiques}

Les données montrent l'ampleur de la morbidité musculosquelettique du membre supérieur et du cou. Près de la moitié de la population enquêtée a déclaré avoir souffert de SMS au cours de l'année écoulée. Une fois sur cinq ces symptômes ont duré au moins 30 jours d'où l'éventualité de passage à la chronicité de cette symptomatologie douloureuse. Les symptômes récents sont retrouvés une fois sur quatre. Une étude algérienne en population de salariés $(n=1750)$ appartenant à différents secteurs d'activités rapporte des prévalences similaires au niveau du membre supérieur [3]. Nos prévalences se rapprochent aussi de celles rapportées par le réseau de surveillance des TMS du membre supérieur des pays de la Loire [15]. La prévalence des SMS au niveau de la nuque était deux fois moins importante que celles rapportées par une études récente $44 \%$, [3]. Cela peut s'expliquer par l'absence du secteur tertiaire dans notre étude. Les prévalences des SMS sur 12 mois varient considérablement entre les études et les différents groupes professionnels [16]. L'étude confirme l'importance de la prévalence sur un an des symptômes de la région de l'épaule qui varie, selon les études, entre 20 à $50 \%$. Elle est moins importante que celle rapportée par l'études algérienne (45\%) [3] et l'étude nationale française 
(41\%) chez les exposés au travail répétitif [17] et correspond aux valeurs rapportées dans la littérature [11].

Les plaintes du poignet sont deux fois moins importantes que celles observées dans l'enquête française sur les TMSms [17] du fait probablement d'un éventail d'entreprises restreint. Elles sont proches de celles rapportées par l'enquête nationale taïwanaise [18].

Au niveau du membre supérieur, la prévalence des SMS est plus élevée chez les femmes. L'emploi des femmes dans les industries sollicitant intensivement les mains et les épaules (électronique et l'habillement) pourrait expliquer cette prédominance. Le cou, l'épaule et le poignet sont les régions les plus touchées à l'exception de la région du coude, qui a concerné plus les hommes. Cela est probablement lié à la nature même du travail qui était différent chez les deux sexes. Cette différence de prévalence de symptômes entre les deux sexes est rapportée par plusieurs enquêtes réalisées en population salariées $[16,19,20]$. La prévalence des SMS augmente avec l'âge pour les deux sexes. Les quinquagénaires sont les plus touchés aussi bien pour le membre supérieur dans son ensemble, que les différentes zones anatomiques y compris le cou, conformément aux données de la littérature $[3,11,5,21]$. Contrairement aux données de l'enquête française [15], la classe 20-29 ans était la moins touchée. Une durée d'exposition relativement courte et un turn-over important notamment dans les entreprises privées pourraient expliquer du moins en partie la faible prévalence des SMS dans cette classe d'âge.

La prévalence des SMS est plus marquée au niveau de l'industrie électronique, la laiterie, l'industrie de l'habillement et l'abattoir de volailles. L'importance de la prévalence des SMS chez les femmes au niveau de l'industrie électronique et de l'habillement est le fait de l'hypersollicitation du membre supérieur en relation avec la nature du travail, ces dernières sont des entreprises à risque de TMS [9]. Dans le reste des entreprises, les hommes sont les plus touchés.

La prévalence des SMS est inversement proportionnelle aux degrés de qualification chez les deux sexes. Ce résultat est probablement lié à la surreprésentation de catégorie ouvrière dans notre échantillon et au fait que cette dernière est une population à haut risque de TMS [9,22].

\section{La prévalence des troubles musculo-squelettiques des membres supérieurs diagnostiqués}

La prévalence des TMSms diagnostiqués est élevée. Plus d'un salarié sur dix présentent au moins un des six principaux TMSms. Elle est proche de celle rapportée par l'étude algérienne (13\%) [3]. La forte prévalence de la pathologie de l'épaule était préoccupante en raison du mauvais pronostic médical et social de cette affection [10,23]. Peu d'études sont disponibles pour estimer la prévalence de l'EPL dans la population générale ou dans la population active [26]. Notre prévalence est similaire à la prévalence décrite dans l'enquête française [14]. L'étude algérienne rapportait une prévalence de $4,5 \%$ [3]. Nos résultats sur la prévalence du STC corroborent les données de la littérature [25]. Les données concernant la prévalence du SCC dans la littérature varient considérablement en fonction des méthodologies utilisées et des secteurs d'activité étudiés $[11,26]$. La prévalence dans la population générale varie entre 5 et $10 \%$ [27]. La prévalence du SCC est semblable à celle rapportée par l'étude française [15]. Les tendinites de l'avant-bras/main sont peu fréquentes comme dans l'étude française, comme celle de De Quervain [15]. Les femmes présentaient plus de TMSms, notamment pour l'épaule et le poignet, comme les plaintes sur les 12 mois et les sept jours chez les femmes au niveau de l'épaule et du poignet. Cette prédominance féminine a été rapportée par plusieurs auteurs $[20,28]$. Elle peut être expliquée par des différences dans l'effet de l'exposition liées au travail physique et les facteurs psychologiques.

L'étude a confirmé l'augmentation de la prévalence des TMSms avec l'âge, pour les deux sexes, conformément aux données de la littérature $[10,11,15]$. L'âge fait intervenir l'ancienneté de l'exposition professionnelle aux facteurs de risque de TMSms, car les salariés les plus âgés sont généralement ceux qui ont les plus grandes durées d'exposition aux contraintes biomécaniques. L'effet de l'âge sur les TMS peut également s'expliquer par des phénomènes dégénératifs des structures péri-articulaires liés au vieillissement, mais aussi par des phénomènes accrus par l'âge d'intolérance à certaines contraintes ou un témoin de la dose cumulée de l'exposition professionnelle (ancienneté d'exposition). Cette accumulation de TMSms chez les travailleurs vieillissant réduit probablement la capacité fonctionnelle et augmente le risque d'invalidité et de licenciement [27].

Nos résultats retrouvent des nettes variations de la prévalence des TMS en fonction des secteurs d'activités et des professions, conformément aux données de la littérature [12]. Les secteurs les plus touchés étaient l'industrie électronique et de l'habillement, notamment, pour les femmes [9]. Les ouvriers non qualifiés de l'industrie sont les plus touchés, Ces professions font appel à des exigences physiques du fait de charges de travail élevées. Elles sont identifiées comme étant à risque élevé de TMSms par des études de prévalence aux États-Unis [9] et d'autres études [15,22]. La prévalence des TMSms est proportionnelle à l'ancienneté au poste actuel notamment chez les femmes après dix ans.

\section{L'exposition aux facteurs de risque de troubles musculo-squelettiques des membres supérieurs}

L'étude montre un niveau élevé d'exposition aux facteurs de risque de TMSms pour la plupart des salariés des deux sexes. Ces scores d'exposition au risque de TMS complètent les données médicales et permettent de dresser une cartographie du risque potentiel des TMSms [11]. Les salariés cumulent en moyenne trois facteurs de risque de TMSms (cou exclu), ce qui 
est considérable du fait du caractère multiplicatif des modèles de risque de TMS $[10,11]$. Les femmes sont nettement surexposées car elles sont majoritaires dans les deux entreprises à risque élevé (l'électronique et l'habillement) [9]. Cette surexposition permet d'expliquer la nette prédominance des SMS et des TMSms chez ces dernières. L'exposition aux facteurs de risque professionnels augmente plus le risque de TMS chez les femmes que les facteurs de risque personnels [24].

La forte exposition des salariés de plus de 50 ans est un résultat préoccupant en raison des difficultés du maintien en emploi [23]. L'intensité de l'exposition est inversement proportionnelle au degré de qualification. L'exposition était particulièrement intense au niveau de l'industrie électronique et d'habillement ce qui pourrait expliquer la prévalence élevée des plaintes et de la pathologie diagnostiquée. Les deux entreprises privées (centre d'enfûtage de gaz et l'imprimerie) sont fortement exposées mais ne présentent pas de pas de pathologies. Un biais de sous-déclaration des plaintes et de la pathologie en vue de la conservation de l'emploi pourrait probablement expliquer cette constatation. Pour le reste, des entreprises, le niveau d'exposition concorde avec les symptômes déclarés et les pathologies diagnostiquées. La prévalence des SMS et des TMSms augmente de manière proportionnelle avec le nombre de facteurs de risque identifiés au poste de travail pour la zone anatomique correspondante, conformément aux données de la littérature [11]. Ces résultats montrent, comme attendu, que les sujets surexposés sont ceux qui rapportent le plus de plaintes et ceux qui présentent des formes avérées de TMSms.

\section{Conclusion}

La présente étude illustre le caractère opérationnel du consensus Saltsa pour la standardisation du recueil des données sur les TMSms au niveau des entreprises algériennes avec la participation des médecins du travail organisés en réseau de surveillance épidémiologique. Elle a souligne l'ampleur de la morbidité musculo-squelettique en termes de plaintes et de pathologies avérées au niveau de nos entreprises. Ces résultats suggèrent déjà la nécessité de la mise en place d'un programme prévention basé sur une intervention multidisciplinaire pour réduire la prévalence des TMSms, d'où la nécessité de la mise en place d'un réseau de surveillance épidémiologique des TMSms en entreprise afin d'enrichir les données qui constitueront une référence sur l'état de santé et l'exposition des salariés par secteur d'activité. Cette étude nous interpelle sur la nécessité d'une meilleure prise en charge des personnes âgées en vue de la réduction de l'exposition aux facteurs de risque et de la préservation de leur l'emploi et la nécessité de réviser les tableaux algériens des maladies professionnelles en vue de leur extension à ces pathologies.

\section{Conflit d'intérêt}

Aucun.

\section{Remerciements}

Avec le support de l'Agence nationale pour le développement de la recherche en santé (ANDRS).

\section{Références}

[1] Derriennic F, Peze M, Davezies P. Analyse de la souffrance dans les troubles musculo-squelettiques : épidémiologie, psychosomatique, médecine du travail. Actes du colloque international de psychodynamique et psychopathologie du travail, Paris 3031 janvier 1997:209-251.

[2] Eurostat. Work And Health In The Eu: a statistical portrait. Data 1994-2002. Luxembourg: Office for official publications of the European communities, 2004, 128 p. http://epp.eurostat. cec.eu.int/offpub/-57-04-807-en.pdf.

[3] Boukerma Z, Hassad S, Tebbal F, Abbassene S, Hamadouche M. TMS ou pathologie d'hypersollicitation musculo-squelettique : étude chez les travailleurs de la région de Sétif. J Med Travail 2007;12:14-20.

[4] Sluiter Jk, Rest Km, Frings-Dresen Mhw. Criteria document for evaluation of the work-relatedness of upper extremity musculoskeletal disorders. Coronel Institute for Occupational and Environmental Health 2000, Amsterdam, rapport, 194 p.

[5] Sluiter JK, Rest KM, Frings-Dresen MHW. Criteria document for evaluation of the work relatedness of upper extremity musculoskeletal disorders. Scand J Work Environ Health 2001; 27(Suppl. 1):1-102.

[6] Ha C, Roquelaure Y. Réseau expérimental de surveillance épidémiologique des troubles musculo-squelettiques dans les Pays de la Loire : protocole de la surveillance dans les entreprises 2002. http://www.invs.sante.fr/publications / 2007/protocole tmsloire/index.html.

[7] Kuorinka I, Jonsson B, Kilbom A, Vinsterberg H, Biering-Sorensen $F$, Andersson $G$, et al. Standardized Nordic questionnaires for the analysis of musculoskeletal symptoms. Appl Ergon 1987;8(3):233-7.

[8] Meyer JP, Frings-Dresen M, Buckle P, Delaruelle D, Privet L, Roquelaure $Y$. Consensus clinique pour le repérage des formes précoces de TMS. Troubles musculo-squelettiques du membre supérieur. Arch Mal Prof 2002;63:32-45.

[9] Silverstein B, Viikari-Juntura E, Kalat J. Use of the prevention index to identify industries at high risk of work-related musculoskeletal disorders of the neck, back, and upper extremities in Washington State, 1990-1998. Am J Ind Med 2002;41:149-69.

[10] Leclerc A. Les TMS du membre supérieur : quelques données épidémiologiques. In: Lasfargues $\mathrm{G}$, Roquelaure $\mathrm{Y}$, Fouquet $\mathrm{B}$, Leclerc A, editors. Pathologie d'hypersollicitation périarticulaires des membres supérieurs. Paris: Masson; 2003. p. 3-26.

[11] Hagberg M, Silverstein B, Wells R. Work related musculoskeletal disorders: a reference book for prevention. London: Taylor \& Francis; 1995. p. 1-421.

[12] Kuorinka I, Jonsson B, Kilbom A, Vinterberg H, Biering-Sorensen $\mathrm{F}$, Andersson $\mathrm{G}$, et al. Analyse des problèmes de l'appareil locomoteur. Questionnaire scandinave. Doc Med Trav 1994;58: $167-70$.

[13] Descatha A, Roquelaure Y, Chastang J.F, Evanoff B, Melchior M, Mariot $C$, et al. Validity of Nordic-style questionnaires in the 
surveillance of upper-limb work-related musculoskeletal disorders. Scand J Work Environ Health 2007;33(1):58-65.

[14] Davis TRC. Diagnostic criteria for upper limb disorders in epidemiological studies. J Hand Surg Br 1998;23B:567-9.

[15] Roquelaure $\mathrm{Y}, \mathrm{Ha} C$, Leclerc A, Touranchet A, Sauteron $M$, Melchior $M$, et al. Epidemiologic surveillance of upper-extremity musculoskeletal disorders in theworking population. Arthritis Rheum 2006;55(5):765-78.

[16] Huisstede BM, Bierma-Zeinstra SM, Koes BW, Verhaar JA. Incidence and prevalence of upper-extremity musculoskeletal disorders. A systematic appraisal of the literature. BMC Musculoskelet Disord 2006;31(7):7.

[17] Leclerc A, Franchi P, Cristofari MF, et al. Affections péri-articulaires des membres supérieurs et organisation du travail. Résultats de l'enquête épidémiologique nationale. Doc Med Travail 1996;65:13-31.

[18] Lee HY, Yeh WY, Chen CW, Wang JD. Prevalence and psychosocial risk factors of upper extremity musculoskeletal pain in industries of Taiwan: a nationwide study. J Occup Health 2005;47(4):311-8.

[19] Treaster DE, Burr D. Gender differences in prevalence of upper extremity musculoskeletal disorders. Ergonomics 2004;47(5): 495-526.

[20] Nordander C, Ohlsson K, Balogh I, Hansson Ga, Axmon A, Persson R, et al. Gender differences in workers with identical repetitive industrial tasks: exposure and musculoskeletal disorders. Int Arch Occup Environ Health 2008;81:939-47.

[21] Holmström E, Engholm G. Musculoskeletal disorders in relation to age and occupation in Swedish construction workers. Am J Ind Med 2003;44(4):377-84.
[22] Melchior M, Roquelaure $\mathrm{Y}$, Evanoff B, Chastang Jf, Ha C Imbernon $\mathrm{E}$, et al. Why are manual workers at high risk of upper limb disorders? The role of physicalwork factors in a random sample of workers in France (the Pays de la Loire study). Occup Environ Med 2006;63(11):754-61.

[23] Roquelaure Y, Cren S, Rousseau F, Touranchet A, Dano C, Fanello $S$, et al. Work status after workers'compensation claims for upper limb musculoskeletal disorders. Occup Environ Med 2004;61:79-81.

[24] Mac Cormack R, Inman R, Wells A. Prevalence of tendinitis and related disorders of the upper extremity in a manufacturing workforce. J Rheumatol 1990;17:958-64.

[25] Descatha A, Leclerc A, Chastang JF, Roquelaure Y, Study Group on Repetitive Work. Incidence of ulnar nerve entrapment at the elbow in repetitive work. Scand J Work Environ Health 2004;30(3):234-40.

[26] Hagberg $M$, Morgenstern H, Kelsh $M$. Impact of occupations and job tasks on the prevalence of carpal tunnel syndrome. Scand J Work Environ Health 1992;18:337-45.

[27] Roquelaure Y, Ha C, Pelier-Cady Mc. Réseau expérimental de surveillance épidémiologique des troubles musculo-squelettiques dans les Pays de la Loire. Surveillance en population générale du syndrome du canal carpien dans le Maine-et-Loire en 2002. Rapport de l'Institut de veille sanitaire, 2004. http:// www.invs.sante.fr/publications/2004/tms131204/rapport_ tms_scc.pdf.

[28] Coury HJCG, Porcatti IA, Alem MER, Oishi J. Influence du sexe sur la survenue des troubles musculo-squelettiques d'origine professionnelle lors des tâches répétitives. Int J Ind Ergonom 2002;29(1):33-9. 